\title{
Macroautophagy without LC3 conjugation?
}

\author{
Cell Research (2017) 27:5-6. doi:10.1038/cr.2016.143; published online 6 December 2016
}

\begin{abstract}
A recent study makes the surprising observation that autophagosomes can still form in the absence of the core conjugation machinery. Furthermore, while such autophagosomes can fuse with lysosomes, their degradation is delayed, and this is associated with delayed destruction of the inner autophagosomal double membrane, highlighting a new role for proteins thought to act exclusively in the formation of autophagosomes in late stages of the autophagic itinerary within autolysosomes.
\end{abstract}

In the last decade, the study of the mechanisms regulating the intertwined connections between autophagosome biogenesis and the lysosomal compartment has gained increasing interest, and has been stimulated by the therapeutic and clinical relevance of this domain [13]. During macroautophagy (hereafter referred to as autophagy), cytoplasmic contents, including damaged organelles, pathogens and aggregate-prone proteins, are sequestered into a doublemembrane structure called autophagosome, which eventually fuses with the lysosome to become an autolysosome, where the degradation occurs [4]. Lysosomal enzymes selectively degrade the inner (but not the outer) autophagosomal membrane (IAM), which finally allows the degradation of the enclosed autophagic content.

The formation of autophagosomes is enabled by more than 30 proteins. Many of these are so-called ATG proteins. Some of these, including ATG3, ATG5, ATG7, ATG10 and ATG12, regulate the conjugation of the ubiquitin-like ATG8 protein family members, such as LC $3 B$, to autophagic precursor membranes enriched in phophatidylethanolamine. These conjugation events are thought to facilitate the extension and closure of the edges of autophagic precursor structure to allow the formation of completed, sealed autophagosomes [5].

In a recent report, Mizushima and collaborators describe how autophagosome-like structures can be generated even in the absence of critical components of the canonical ATG conjugation systems, namely ATG3 [6]. Strikingly, even though these proteins are required for sealing of autophagosomes, these ATG proteins do not appear to be essential for autolysosome formation but are important for efficient degradation of the IAM.

To further characterize the autophagosome maturation step, the authors relied on the autophagosomal SNARE syntaxin17 (STX17), whose hairpintype tail-anchor is essential for targeting and driving the fusion of mature autophagosomes to the endosomal/ lysosomal compartment [7]. Using live imaging, the authors were able to identify and discriminate four steps during autophagosome maturation, namely: STX17 recruitment, lysosomal fusion, IAM degradation and STX17 release. Of particular interest, STX17 recruitment induces an elliptical-to-spherical transition, which is likely coincident with the fission between the outer and inner autophagosomal membranes (Figure 1).

As expected, the rate of successful autophagosome formation was substantially reduced in ATG3 knockout (KO) cells, confirming that the ATG conjugation systems are indeed important for the late stages of autophagosome forma- tion $[8,9]$. Consistent with this observation, autophagic substrate degradation was dramatically impaired in such cells. Nevertheless, the STX17-positive autophagosome-like structures in ATG3 $\mathrm{KO}$ cells were positive for SNAP29 and capable of undergoing acidification and co-localizing with the late endosome/ lysosome marker, LAMP1. Thus, the fusion between autophagosome-like structures and lysosomes occur almost normally in ATG3 KO cells, as opposed to wild-type (WT) cells, although at a lower rate. Interestingly, their experiments suggested that IAM degradation is significantly delayed in ATG conjugation-deficient cells. This phenomenon was confirmed by immuno-electron microscopy and was not due to a lysosomal defect. Their data also suggested that the collapse of the IAM triggers the immediate dissociation of STX17 from the autolysosomes. In addition, they observed that a higher proportion of the STX17-positive autophagosomelike structures were elliptic in ATG3 KO cells, as opposed to those in WT cells. These results also suggest that, despite the STX17 recruitment, the ultimate fission step is somehow impaired in ATG non-competent cells (Figure 1).

To summarize, the function of ATG proteins had been investigated previously almost exclusively in the context of autophagosome formation. These novel findings reveal a functional role of ATG proteins involved in ATG8 family conjugation in late stages of autophagy after autophagosome-lysosome fusion. The ability to form some type of autophagic structures in the absence of these proteins is consistent with previous observations that a small propor- 


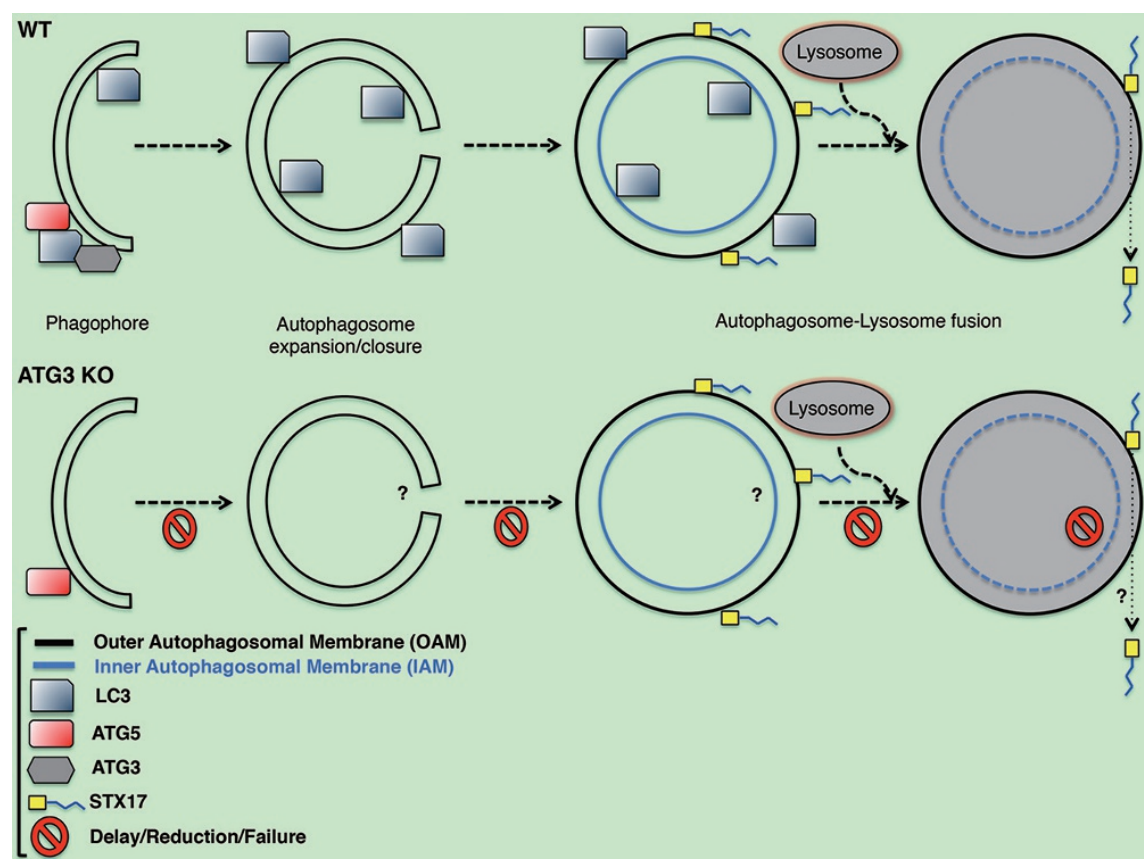

Figure 1 IAM route to lysosomal degradation depends on ATG proteins. The function of ATG proteins has been mainly investigated in the context of autophagosome formation. In normal conditions, the IAM of WT cells is sensitive to lysosomal degradation. While autophagic activity is strongly suppressed in ATG conjugationdeficient cells (exemplified by ATG3 KO cells in this Figure), autophagosome-like structures can still form and acquire STX17. These syntaxin17-positive structures are able to fuse with lysosomes. Nevertheless, degradation of the IAM is significantly impaired. These findings reveal a novel function of ATGs with regard to autophagosome maturation steps.

tion of autophagosome-like structures can mature into autolysosomes in ATG conjugation-deficient cells. This may explain, at least in part, some of the phenotypic differences and severities existing among different autophagy KO mouse models, depending whether appears to be compromised [13].

\section{Maurizio Renna ${ }^{1}$, David C Rubinsztein ${ }^{1}$}

${ }^{I}$ Department of Medical Genetics, University of Cambridge, Cambridge Institute for Medical Research, Wellcome Trust/MRC Building, Addenbrooke's Hospital, Hills Road, Cambridge, CB2 2XY, UK

Correspondence: David C Rubinsztein

E-mail: dcr1000@hermes.cam.ac.uk

\section{References}

1 Mizushima N, Levine B, Cuervo AM, et al. Nature 2008; 451:1069-1075.

2 Ravikumar B, Sarkar S, Davies JE, et al. Physiol Rev 2010; 90:1383-1435.

3 Mizushima N, Komatsu M. Cell 2011; 147:728-741.

4 Bento CF, Renna M, Ashkenazi A, et al. Annu Rev Biochem 2016; 85:685-713.

5 Ktistakis NT, Tooze SA. Trends Cell Biol 2016; 26:624-635.

6 Tsuboyama K, Koyama-Honda I, Sakamaki Y, et al. Science 2016; 354:1036-1041.

7 Itakura E, Kishi-Itakura C, Mizushima N. Cell 2012; 151:1256-1269.

8 Fujita N, Hayashi-Nishino M, Fukumoto H, et al. Mol Biol Cell 2008; 19:4651-4659.

9 Noda T, Fujita N, Yoshimori T. Cell Death Differ 2009; 16:984-990.

10 Komatsu M, Waguri S, Ueno T, et al. J Cell Biol 2005; 169:425-434.

11 Malhotra R, Warne JP, Salas E, et al. Autophagy 2015; 11:145-154.

12 Saitoh T, Fujita N, Jang MH, et al. Nature 2008; 456:264-268.

13 Nguyen TN, Padman BS, Usher J, et al. J Cell Biol 2016 Nov 18. doi: 10.1083/ jcb.201607039 